\title{
Asociación entre clima organizacional y motivación de enfermeras en un hospital de la Policía Nacional del Perú
}

\author{
Association between organizational climate and motivation of nurses in a hospital of the National Police of \\ Peru
}

Ana Sheyla Porras-Balvin ${ }^{1,2, a, b}$, Juana Elena Durand-Barreto 2,a,c

\section{RESUMEN}

Objetivo: Identificar la asociación del clima organizacional y la motivación del personal de enfermería en centro quirúrgico del Hospital Luis N. Sáenz. Material y métodos: El presente estudio es cuantitativo, descriptivo y transversal. La población la conformaron 30 enfermeras. Se validaron 2 formularios para medir clima organizacional y motivación obteniendo $\alpha$ de Crombach de 0,95 y 0,92 , respectivamente. El análisis principal de asociación se realizó mediante Ji cuadrado. Resultados: La población se caracterizó por ser del sexo femenino $(86,67 \%)$ y laborar más de 20 años $(46,67 \%)$ en la institución. El clima organizacional favorable es de $50 \%$ y motivación alta en $53 \%$. Entre las dimensiones del clima organizacional favorables fueron: la supervisión (56,67\%), comunicación $(46,67 \%)$ y realización personal $(46,67 \%)$. En la motivación alta, las dimensiones que resaltaron fueron, logros $(56,67 \%)$, promoción $(43,33 \%)$ y reconocimiento $(43,33 \%)$. La asociación entre las variables clima organizacional y motivación fue significativa $(\mathrm{p}=0,05)$. Conclusiones: Existe asociación entre clima organizacional y motivación enfermeras en un hospital de la Policía Nacional del Perú, por lo que sería necesario plantear investigaciones de intervención para mejorar la gestión del recurso humano enfermero en organizaciones castrenses.

PALABRAS CLAVE: Administración de personal en hospitales, motivación, Enfermería de quirófano.

\section{SUMMARY}

Objective: To identify the association of the organizational climate and the motivation of the nursing staff in the surgical center of the Hospital Luis N. Saenz. Material and methods: The present study is quantitative, descriptive and transversal. The population consisted of 30 nurses. Two forms were validated to measure organizational climate and motivation, obtaining Crombach's $\alpha$ of 0.95 and 0.92 , respectively. The main association analysis was performed using Chi square. Results: The population was characterized by being female (86.67\%) and working more than 20 years $(46.67 \%)$ in the institution. The favorable organizational climate is $50 \%$ and high motivation in $53 \%$. Among the dimensions of the favorable organizational climate were: supervision $(56.67 \%)$, communication $(46.67 \%)$ and personal fulfillment (46.67\%). In high motivation, the dimensions that stood out were, achievements $(56.67 \%)$, promotion $(43.33 \%)$ and recognition $(43.33 \%)$. The association between the variables organizational climate and motivation was significant $(\mathrm{p}=0.05)$. Conclusions: There is an association between the organizational climate and the motivation of nurses in a hospital of the National Police of Peru, so it would be necessary to propose intervention researches to improve the management of the nursing human resource in military organizations.

KEYWORDS: Personnel administration hospital, motivation, operating room nursing.

Hospital Luis Nicasio Sáenz. Lima, Perú.

Universidad Nacional Mayor de San Marcos, Lima, Perú.

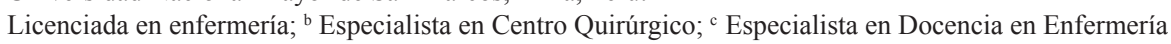




\section{INTRODUCCIÓN}

Para la gestión de manejo del personal en las instituciones y/o organizaciones, se debe determinar mecanismos de planificación en los recursos humamos. Los jefes o administradores tendrán en cuenta y motivarán al personal para explotar sus habilidades y destrezas para cumplir de forma efectiva con la gran demanda de atención de salud (1).

El clima organizacional se define como el conjunto de atributos que pueden ser percibidos acerca de una organización particular y/o sus subsistemas, y que puede ser inducido por la forma en que la organización interactúa con sus miembros y con su ambiente (2).

Así mismo, la motivación puede ser definida como el proceso de planificación y realización de una serie de actividades y comportamientos dirigidos a la obtención de un objetivo que nos sirva para satisfacer un motivo inicial (3).

Ambas variables, clima organizacional y motivación, han sido estudiadas en contextos como en el país de Paraguay, donde identificaron la percepción del clima organizacional en sus diversas dimensiones del personal sanitario y administrativo, fue un estudio observacional, descriptivo de corte transversal con componente analítico. Teniendo como resultados niveles insatisfactorios en todas sus dimensiones estudiadas relacionadas al clima organizacional en el personal del hospital Militar (4).

Asímismo,enBrasilevaluaron elclima organizacional en instituciones policiales militares del estado de Santa Clara, dirigido al personal exclusivamente militar, mediante un estudio exploratorio de caso, a través de metodología cualitativa focus group, encontraron como resultados, que los militares se sienten tensionados en relación a diversas variables del clima organizacional (5).

En este contexto, el Hospital de la Policía Nacional del Perú, tutelado por el Ministerio del interior, en plan de cubrir las necesidades de salud del personal policial (personal en actividad, retirados y familiares directos) en determinados tiempo, incluye personal nuevo para cubrir su propia demanda de salud policial, el cual a su vez, se rige por el reglamento institucional(6), por lo que el clima organizacional y la motivación son, dos indicadores fundamentales para el manejo de sus recursos, siendo relevante conocer la relación que pueda existir entre ellos para mantener una fuerza laboral, estable y dinámica.
Al no existir estudios nacionales previos en el contexto policial y/o militar, se ve conveniente desarrollar un estudio en el Hospital Luis N. Sáenz - PNP sobre el personal de enfermería que labora en centro quirúrgico para determinar el tipo de clima organizacional al cual están sometidos y sobre la motivación con la que cuentan diariamente para desenvolverse en el trabajo de manera óptima. Los resultados del estudio están orientados a conocer el contexto laboral en instituciones castrenses como a nivel institucional para proporcionar información actualizada que permita reflexionar al personal de enfermería y a la jefatura del servicio, para elaborar proyectos de mejora a corto y largo plazo.

\section{MATERIAL Y MÉTODOS}

La investigación es cuantitativa, descriptiva y correlacional de corte transversal. Se realizó en el Hospital Luis Nicasio Sáenz - PNP, Lima-Perú. La población estuvo conformada por 30 enfermeras del centro quirúrgico, teniendo en cuenta, criterios de inclusión a los profesionales de enfermería de ambos sexos, de procedencia civil y asimilados con grado de oficial, laborando más de 1 año en dicho servicio.

La técnica utilizada fue la encuesta, y los instrumentos estuvieron conformadas para la medición del clima organizacional por la Escala de Clima Laboral (CL-SPC) de Carrillo(7), el cual fue modificado para adaptarlo a la realidad, conformando al final solo 40 ítems sin modificar las 5 dimensiones originales (realización personal, involucramiento laboral, supervisión, comunicación y condiciones laborales).

Asimismo, para la variable motivación se fundamentó en las teorías de motivación de Herzberg y se utilizó la Escala de Likert adaptada por Vásquez (8). El mismo que fue modificado con 2 ítems adicionales en cada dimensión, obteniéndose 25 ítems, sin embargo se mantuvo las 5 dimensiones del constructo teórico original (reconocimiento, logros, posibilidad de crecimiento, promoción y responsabilidad). Ambos cuestionarios fueron validados por juicio de expertos, conformado por especialistas en el área y procesados en tablas de concordancia y prueba binomial. De la misma manera, se aplicó la prueba piloto de los instrumentos y se determinó la validez estadística del clima organizacional a través de la prueba de confiabilidad mediante $\alpha$ de Crombach con $\alpha=0.95$ y para motivación $\alpha=0,92$. 
Los datos recolectados fueron procesados mediante el paquete estadístico de Excel 2013, previa elaboración de la tabla de códigos y matriz de datos. Los resultados fueron presentados en tablas y gráficos estadísticos para su análisis e interpretación considerando el marco teórico. Para la medición de la variable se utilizó la estadística descriptiva (porcentajes, la frecuencia absoluta, y promedio aritmético. La variable clima organizacional se valoró en favorable y desfavorable. La variable motivación se categorizó en alta y baja. Para comprobar la hipótesis de estudio se aplicó la prueba no paramétrica del Ji - cuadrado con un nivel de significancia del 95\%. El proyecto tuvo aprobación del Comité de Ética en investigación del Complejo Hospitalario PNP-LNS.

Se consideró aplicación de consentimiento informado y se respetó los principios éticos morales de los participantes, resguardando el anonimato de los datos recolectados.

\section{RESULTADOS}

La población del estudio se caracteriza en su mayoría por tener entre 38 a 42 años de edad (36,67\%), de sexo femenino (86,67\%), tener más de 20 años de servicio $(46,67 \%)$ y ser oficiales de servicio $(76,67 \%)$ (tabla 1).

Tabla 1. Datos generales de profesionales de enfermería en un Hospital- PNP.

\begin{tabular}{llcc}
\hline \multicolumn{1}{c}{ Datos } & \multicolumn{1}{c}{ Categoría } & n & \multicolumn{1}{c}{$\%$} \\
\hline \multirow{4}{*}{ Edad } & $28-32$ años & 4 & 13,33 \\
& $33-37$ años & 2 & 6,67 \\
& $38-42$ años & 11 & 36,67 \\
& $43-47$ años & 8 & 26,67 \\
Estado Civil & $48-55$ años & 5 & 16,66 \\
& Soltero & 14 & 46,67 \\
& Casado & 14 & 46,67 \\
Sexo & Divorciado & 1 & 3,33 \\
& Viudo & 1 & 3,33 \\
Tiempo de servicio & Masculino & 04 & 13,33 \\
& Femenino & 26 & 86,67 \\
\multirow{5}{*}{ Condición laboral 20 años } & 9 & 30,00 \\
& $>$ 20 años & 7 & 23,33 \\
& Oficial de servicios & 23 & 46,67 \\
& Civil & 7 & 23,33 \\
\hline
\end{tabular}

El $50 \%$ de enfermeros expresó que el clima organizacional es favorable, destacando la dimensión supervisión (56,67\%) (tabla 2). Como ítems destacados en cada dimensión tenemos: Las actividades en la que se trabaja me permiten aprender y desarrollarme $(70 \%)$, se sienten comprometidos con la institución (100\%), las responsabilidades del puesto están claramente definidas $(96,67 \%)$, es posible la interacción con las personas de mayor jerarquía $(73,33 \%)$, y el personal tiene la oportunidad de tomar decisiones en torno a sus responsabilidades (70\%). Los aspectos referidos a desfavorables $(50 \%)$ se atribuye a que los supervisores no expresan reconocimiento por sus logros $(86.67 \%)$, no hay una clara definición de la visión, misión y valores en el servicio (60\%), el trato no es justo en la institución $(86,67 \%)$, no se conocen los avances en otras áreas del servicio (70\%) y la administración de los recursos no es buena $(83,33 \%)$ (tabla 3$)$.

Respecto a la motivación, el 53\% obtuvo motivación alta y el $47 \%$ baja. Sobresaliendo en la motivación alta; la dimensión logros $(56,67 \%)$ (tabla 4). El detalle de las 5 categorías se puede apreciar en la tabla 5, como ítems más destacados en cada dimensión se obtiene, el personal manifiestan que el trabajo que realizan es valorado por el jefe inmediato $(43,33 \%)$, las actividades que desempeño corresponden a mi función (90\%), participo en la toma de decisiones dentro de mi sala $(83,33 \%)$, trabajan organizadamente en equipo (86,6\%), y cumplen el horario establecido y registran puntualidad (93,33\%); a la vez se incluye en la motivación baja, no reciben de la institución o jefe del servicio algún reconocimiento documentado durante el último año $(93,33 \%)$, en algunas ocasiones consideran que los resultados que le ofrecen en el

Tabla 2. Clima Organizacional según dimensiones del profesional de enfermería en un Hospital- PNP.

\begin{tabular}{|c|c|c|c|c|}
\hline \multirow{2}{*}{ Dimensiones } & \multicolumn{2}{|c|}{ Favorable } & \multicolumn{2}{|c|}{ Desfavorable } \\
\hline & $\mathrm{n}$ & $\%$ & $\mathbf{n}$ & $\%$ \\
\hline $\begin{array}{l}\text { Realización } \\
\text { personal }\end{array}$ & 14 & $46,67 \%$ & 16 & $53,33 \%$ \\
\hline $\begin{array}{l}\text { Involucramiento } \\
\text { Laboral }\end{array}$ & 15 & $50,00 \%$ & 15 & $50,00 \%$ \\
\hline Supervisión & 17 & $56,67 \%$ & 13 & $43,33 \%$ \\
\hline Comunicación & 14 & $46,67 \%$ & 16 & $53,33 \%$ \\
\hline $\begin{array}{l}\text { Condición } \\
\text { Laboral }\end{array}$ & 11 & $36,67 \%$ & 19 & $63,33 \%$ \\
\hline
\end{tabular}


Tabla 3. Nivel de Clima Organizacional del Profesional de Enfermería Según Dimensiones de un Hospital -PNP.

Ítems

\begin{tabular}{cccc}
\multicolumn{2}{c}{ favorable } & \multicolumn{2}{c}{ desfavorable } \\
\hline n & $\%$ & n & $\%$
\end{tabular}

Realización personal

Existen oportunidades de progresar en la institución

15

El jefe se interesa por el éxito de los colaboradores

Participo y defino los objetivos y las acciones para lograrlo.

Se valora los altos niveles de desempeño.

Los supervisores expresan reconocimiento por los logros.

Las actividades en la que se trabaja me permiten aprender y desarrollarme.

Los jefes promueven la capacitación que necesito.

La institución promueve mi desarrollo personal.

Involucramiento laboral

Me siento comprometido con el éxito del centro quirúrgico

Cada profesional asegura sus niveles de logro en el trabajo

Cada profesional se considera factor clave para el éxito del servicio.

Me siento comprometido con la institución.

Cumplir con las actividades diarias en el servicio, me permite desarrollo personal.

Cumplir con las actividades laborales es una tarea estimulante.

Las áreas del servicio son motivo de orgullo para mí.

Hay clara definición de visión, misión y valores en el servicio.

Supervisión

Los jefes brindan apoyo para superar los obstáculos que se presentan.

En el servicio, se mejoran continuamente los métodos de trabajo.

Las responsabilidades del puesto están claramente definidas.

Se dispone de un sistema para el seguimiento y control de actividades.

Existen normas y procedimientos como guías de trabajo.

Los objetivos del trabajo están claramente definidos

El trabajo se realiza en función a guías y protocolos establecidos.

Existe un trato justo en la institución.

Comunicación

Encuentro acceso a la información necesaria para cumplir con mi trabajo.

En mi quirófano, la información fluye adecuadamente.

En los grupos de trabajo, existe una relación armoniosa.

Es posible la interacción con las personas de mayor jerarquía.

El servicio promueve la comunicación interna.

La jefa escucha los planteamientos que se le hace.

Existe colaboración entre el personal de las diversas salas.

5

15

21

8

4

21

5

$21 \quad 70,00 \%$

$16,67 \%$

$16,67 \%$

5

$\begin{array}{cc}50,00 \% & 15 \\ 50,00 \% & 15 \\ 70,00 \% & 9 \\ 26,67 \% & 22 \\ 13,33 \% & 26 \\ 70,00 \% & 9\end{array}$

$\begin{array}{cccc}30 & 100,00 \% & 0 & 0,00 \% \\ 25 & 83,33 \% & 5 & 16,67 \% \\ 24 & 80,00 \% & 6 & 20,00 \%\end{array}$

$30 \quad 100,00 \% \quad 0 \quad 0,00 \%$

$30 \quad 100,00 \% \quad 0 \quad 0,00 \%$

$\begin{array}{llll}26 & 86,67 \% & 4 & 13,33 \%\end{array}$

$19 \quad 63,33 \% \quad 11 \quad 36,67 \%$

$12 \quad 40,00 \% \quad 18 \quad 60,00 \%$

\begin{tabular}{cccc}
13 & $43,33 \%$ & 17 & $56,67 \%$ \\
14 & $46,67 \%$ & 16 & $53,33 \%$ \\
29 & $96,67 \%$ & 1 & $3,33 \%$ \\
13 & $43,33 \%$ & 17 & $56,67 \%$ \\
16 & $53,33 \%$ & 14 & $46,67 \%$ \\
18 & $60,00 \%$ & 12 & $40,00 \%$ \\
17 & $56,67 \%$ & 13 & $43,33 \%$ \\
4 & $13,33 \%$ & 26 & $86,67 \%$ \\
\hline
\end{tabular}

$\begin{array}{cccc}12 & 40,00 \% & 18 & 60,00 \% \\ 18 & 60,00 \% & 12 & 40,00 \% \\ 15 & 50,00 \% & 15 & 50,00 \% \\ 22 & 73,33 \% & 8 & 26,67 \% \\ 19 & 63,33 \% & 11 & 36,67 \% \\ 21 & 70,00 \% & 9 & 30,00 \% \\ 13 & 43,33 \% & 17 & 56,67 \%\end{array}$




\begin{tabular}{|c|c|c|c|c|}
\hline \multirow{2}{*}{ Ítems } & \multicolumn{2}{|c|}{ favorable } & \multicolumn{2}{|c|}{ desfavorable } \\
\hline & $\mathbf{n}$ & $\%$ & $\mathbf{n}$ & $\%$ \\
\hline Se conocen los avances en las otras áreas del servicio. & 9 & $30,00 \%$ & 21 & $70,00 \%$ \\
\hline \multicolumn{5}{|l|}{ Condición laboral } \\
\hline Los compañeros de trabajo cooperan entre sí. & 15 & $50,00 \%$ & 15 & $50,00 \%$ \\
\hline $\begin{array}{l}\text { El personal tiene la oportunidad de tomar decisiones en tarea } \\
\text { de sus responsabilidades. }\end{array}$ & 21 & $70,00 \%$ & 9 & $30,00 \%$ \\
\hline $\begin{array}{l}\text { El grupo con el que trabajo, funciona como un equipo bien } \\
\text { integrado. }\end{array}$ & 15 & $50,00 \%$ & 15 & $50,00 \%$ \\
\hline $\begin{array}{l}\text { Cuento con la oportunidad de realizar el trabajo lo mejor que } \\
\text { puedo. }\end{array}$ & 20 & $66,67 \%$ & 10 & $33,33 \%$ \\
\hline Existe buena administración de los recursos. & 5 & $16,67 \%$ & 25 & $83,33 \%$ \\
\hline $\begin{array}{l}\text { La remuneración es atractiva en comparación con la de otras } \\
\text { instituciones. }\end{array}$ & 12 & $40,00 \%$ & 18 & $60,00 \%$ \\
\hline $\begin{array}{l}\text { Los objetivos del personal guardan relación con la visión de } \\
\text { la institución. }\end{array}$ & 13 & $43,33 \%$ & 17 & $56,67 \%$ \\
\hline Dispongo de tecnología que facilite mi trabajo. & 9 & $30,00 \%$ & 21 & $70,00 \%$ \\
\hline
\end{tabular}

Tabla 4. Motivación según dimensiones del profesional de enfermería en un Hospital- PNP.

\begin{tabular}{lcccc}
\hline \multirow{2}{*}{\multicolumn{1}{c}{ Dimensiones }} & \multicolumn{2}{c}{ Alta } & \multicolumn{2}{c}{ Baja } \\
\cline { 2 - 5 } & n & $\mathbf{\%}$ & $\mathbf{n}$ & $\mathbf{\%}$ \\
\hline Reconocimiento & 13 & $43,33 \%$ & 17 & $56,67 \%$ \\
Logros & 17 & $56,67 \%$ & 13 & $43,33 \%$ \\
Posibilidad de crecimiento & 15 & $50,00 \%$ & 15 & $50,00 \%$ \\
Promoción & 13 & $43,33 \%$ & 17 & $56,67 \%$ \\
Responsabilidad & 10 & $33,33 \%$ & 20 & $66,67 \%$ \\
\hline
\end{tabular}

Tabla 5. Nivel de Motivación del Profesional de Enfermería Según Dimensiones de un Hospital -PNP.

\begin{tabular}{|c|c|c|c|c|}
\hline \multirow{2}{*}{ Ítems } & \multicolumn{2}{|c|}{ Alta } & \multicolumn{2}{|c|}{ Baja } \\
\hline & $\mathbf{n}$ & $\%$ & n & $\%$ \\
\hline \multicolumn{5}{|l|}{ Reconocimiento } \\
\hline En mi institución reconocen habitualmente la buena labor que realiza el servicio. & 4 & $13,33 \%$ & 26 & $86,67 \%$ \\
\hline Los premios y reconocimientos son distribuidos en forma justa. & 3 & $10,00 \%$ & 27 & $90,00 \%$ \\
\hline El trabajo que realizo es valorado por mi jefe inmediato. & 13 & $43,33 \%$ & 17 & $56,67 \%$ \\
\hline $\begin{array}{l}\text { Ha recibido de la institución o jefe del servicio algún reconocimiento documentado } \\
\text { durante el último año. }\end{array}$ & 2 & $6,67 \%$ & 28 & $93,33 \%$ \\
\hline Mis compañeros de trabajo valoran y reconocen mi trabajo. & 8 & $26,67 \%$ & 22 & $73,33 \%$ \\
\hline \multicolumn{5}{|l|}{ Logros } \\
\hline Mi trabajo contribuye directamente el alcance de los objetivos. & 26 & $86,67 \%$ & 4 & $13,33 \%$ \\
\hline Considero que los resultados que me ofrecen en mi trabajo son los adecuados. & 13 & $43,33 \%$ & 17 & $56,67 \%$ \\
\hline Me siento a gusto de formar parte de la institución. & 25 & $83,33 \%$ & 5 & $16,67 \%$ \\
\hline El trabajo que realizo permite que desarrolle al máximo todas mis capacidades. & 27 & $90,00 \%$ & 3 & $10,00 \%$ \\
\hline
\end{tabular}




\begin{tabular}{|c|c|c|c|c|}
\hline \multirow{2}{*}{ Ítems } & \multicolumn{2}{|c|}{ Alta } & \multicolumn{2}{|c|}{ Baja } \\
\hline & n & $\%$ & n & $\%$ \\
\hline Las tareas que desempeño corresponden a mi función. & 27 & $90,00 \%$ & 3 & $10,00 \%$ \\
\hline \multicolumn{5}{|l|}{ Posibilidad de crecimiento } \\
\hline La institución me motiva para poder alcanzar un puesto mejor al que usted tiene. & 4 & $13,33 \%$ & 26 & $86,67 \%$ \\
\hline $\begin{array}{l}\text { Considero que la distribución física del área donde laboro me permite trabajar } \\
\text { cómoda y eficientemente. }\end{array}$ & 9 & $30,00 \%$ & 21 & $70,00 \%$ \\
\hline Me dan la libertad para hacer mi trabajo de acuerdo a mi criterio & 17 & $56,67 \%$ & 13 & $43,33 \%$ \\
\hline Participo en la toma de decisiones dentro de mi sala. & 25 & $83,33 \%$ & 5 & $16,67 \%$ \\
\hline Me entregan la formación necesaria para desarrollarme como persona y profesional. & 17 & $56,67 \%$ & 13 & $43,33 \%$ \\
\hline \multicolumn{5}{|l|}{ Promoción } \\
\hline Me interesa el desarrollo del servicio. & 29 & $96,67 \%$ & 1 & $3,33 \%$ \\
\hline Me siento comprometido con mi institución y con el servicio donde laboro. & 29 & $96,67 \%$ & 1 & $3,33 \%$ \\
\hline Participó activamente en las actividades y reuniones programadas en el servicio. & 19 & $63,33 \%$ & 11 & $36,67 \%$ \\
\hline En mi institución existen oportunidades de promociones y ascensos. & 15 & $50,00 \%$ & 15 & $50,00 \%$ \\
\hline Trabaja organizadamente en equipo & 26 & $86,67 \%$ & 4 & $13,33 \%$ \\
\hline \multicolumn{5}{|l|}{ Responsabilidad } \\
\hline Cumplo con el horario establecido y registro puntualidad. & 28 & $93,33 \%$ & 2 & $6,67 \%$ \\
\hline Promuevo desarrollo en beneficio del servicio. & 22 & $73,33 \%$ & 8 & $26,67 \%$ \\
\hline Actúa de cuerdo a normas, guías y protocolos establecidos. & 23 & $76,67 \%$ & 7 & $23,33 \%$ \\
\hline Cumplo con las tareas encomendadas & 28 & $93,33 \%$ & 2 & $6,67 \%$ \\
\hline $\begin{array}{l}\text { Asumo con responsabilidad las tareas asignadas por el jefe del servicio y jefe } \\
\text { inmediato superior }\end{array}$ & 28 & $93,33 \%$ & 2 & $6,67 \%$ \\
\hline
\end{tabular}

trabajo no son adecuados (57\%), la institución no motiva para poder alcanzar un puesto mejor $(86,67 \%)$, no existen oportunidades de promociones y ascensos $(50 \%)$, y no siempre promueven el desarrollo en beneficio del servicio $(26,67 \%)$.

\section{DISCUSIÓN}

El presente estudio tuvo como objetivo, determinar la asociación entre clima organizacional y la motivación del profesional de enfermería del Centro Quirúrgico de un hospital de la PNP, encontrando significativamente motivación alta en el clima favorable, determinando que probablemente existiría asociación entre ambas variables.

El clima organizacional y la motivación constituyen dos temas de gran interés en la psicología organizacional, por sus implicancias en la productividad del recurso humano en ambientes laborales, constituyendo los indicadores de la calidad de vida en el trabajo.
Sarmiento, con su estudio llegó a comprobar que el Clima Organizacional se relaciona directamente con la Motivación Laboral en una empresa de servicio de Agua potable en la ciudad de Trujillo, esta observó que el $77 \%$ del personal administrativo está de acuerdo con el clima organizacional, obteniendo un promedio del $70 \%$ de los trabajadores se sienta motivado (9).

También coincide con la investigación de Paredes, al encontrar relación significativa alta entre la motivación personal y el clima organizacional en el personal de obstetricia en un hospital de la ciudad de Huancayo (10).

Respecto a enfermería, este estudio identificó que el clima organizacional es favorable solo en $50 \%$, esto podría conllevar a interferir en el logro de objetivos personales, profesionales e institucionales y por ende en la satisfacción laboral. En comparación de Huaranga, quién también realizó investigación en profesionales de enfermería del Hospital de los Olivos, halló que el $72.4 \%$ refiere clima organizacional desfavorable 
(11). Consideramos a éste, un resultado inquietante, porque muestra un impacto alto a diferencia de esta investigación.

Respecto a ello Bernal y Col, identificaron lo siguiente; el ambiente laboral donde se desarrollan los procesos de salud se ve influenciado por el clima organizacional prevaleciente en las instituciones hospitalarias, de tal forma un ambiente óptimo, favorecería el entorno de trabajo y con ello, el compromiso y desempeño de los empleados (12).

Una de las dimensiones más resaltantes es la supervisión, porque obtiene resultados en su mayoría favorables, quees determinadapor las responsabilidades del puesto que están claramente definidas, el trabajo se realiza en función a guías y protocolos. A diferencia de la investigación de Leandro, éste afirma que sólo el $37 \%$ de los trabajadores consideran supervisión adecuada, alegando que los jefes no promueven la capacitación que necesitan y que el director General, muestra poco interés en el éxito de los trabajadores (13).

Otra dimensión es el involucramiento laboral, con el que se obtiene resultados favorables en $50 \%$, lo cual podría atribuirse a que los profesionales manifiestan estar comprometidos con el éxito de las cirugías, pero aún les falta disposición e interés en el desarrollo de actividades diarias. Para Rosales, según sus resultados para el involucramiento laboral el $34,9 \%$ del personal presenta un nivel inadecuado y el 43\%a un nivel regular. De esta manera se podría mencionar que para el involucramiento laboral tiene que ver mucha la actitud del personal (14).

La motivación en la enfermería, es la pasión que tiene el profesional para prestar servicios de salud, y el factor económico no siempre será un principal determinante para motivar, existen otros tipos de incentivos igualmente o más eficaces que sólo conseguir reconocimiento económico. Entre ellas está el reconocimiento profesional, la satisfacción personal, la participación en la toma de decisiones, desarrollo profesional, etc.

Llagas define que la motivación es el deseo de entregarse al máximo para obtener las metas de la organización, determinado por la satisfacción de alguna necesidad individual. Si bien la motivación general, se refiere al esfuerzo por conseguir todo objetivo, nos enfocamos en metas organizacionales a fin de reflejar nuestro principal interés, por el comportamiento conexo con la motivación y el sistema de valores que rige la organización (15).

Se obtuvo como resultado, Motivación alta en el profesional de enfermería, quienes expresan que el trabajo, les permite desarrollo al máximo de todas sus capacidades, obteniendo libertad para realizar sus actividades de acuerdo a propio criterio, su trabajo contribuye directamente en el logro de objetivos, y se interesa por el desarrollo del servicio. Esta motivación alta, es alentadora a diferencia de otros estudios, porque obtienen resultados medios y bajos. Como Lucas, quién concluyó que el nivel de motivación del personal de Enfermería es medio, por déficit de los recursos y por la poca estrategia de los líderes, para mejorar el interés y satisfacción del personal en el desarrollo de sus labores" (16).

La investigación Motivación y Satisfacción laboral del profesional enfermero del hospital Ramón Castilla - EsSalud, obtuvo como resultados el 57\% donde las enfermeras presentan motivación media. Al analizar dicha variable se encontró que las dimensiones, importancia, retroalimentación y autonomía presentan niveles medio con tendencia alta, mientras que las dimensiones identidad y variedad de la tarea, presentan mayoritariamente nivel bajo (17).

Rosales, encuentra en su investigación, nivel motivacional medio en el personal de enfermería del servicio de emergencia de un hospital público de Lima (14). Dichas investigaciones obtienen resultados medios y bajos. Por lo tanto, la motivación es un indicador personal, que podría ser afectado por la rutina, estrés o problemas internos que vive cada persona, y muchas veces es olvidado por las organizaciones.

En esta investigación, una de las dimensiones de la motivación es el reconocimiento, que resultó ser desfavorable y caracterizada porque durante el último año, los profesionales no han recibido reconocimiento por la institución, estos resultados coinciden con los de Vásquez, ella determina que entre los factores motivacionales, se encuentra la falta de reconocimiento por parte de la Dirección del hospital. Los trabajadores carecen de incentivos, carta de felicitación, beca de capacitaciones, entre otros alicientes; tal es así que el $45,2 \%$ está muy en desacuerdo y el $35,7 \%$ afirma estar en desacuerdo (8). Entonces encontramos que el reconocimiento es un factor muy valorado para los trabajadores de toda institución, pues en ella ven reflejado los resultados de su esfuerzo. Observamos 
que las entidades hospitalarias se preocupan por la productividad en la atención de pacientes, más no se enfocan en motivar al personal para que pueda desempeñarse con mayor entrega y compromiso.

Debido a esto, es fundamental promover las condiciones necesarias mediante la formulación de estrategias, orientado a fortalecer el clima organizacional que contribuya en la motivación del personal del centro quirúrgico, las cuales contribuyen a optimizar los recursos y capacidades del sistema de salud, repercutiendo en la satisfacción laboral del usuario interno y mejorando la calidad de atención al usuario externo. Siendo necesario realizar mediciones periódicas para seguir fortaleciendo aquellas deficiencias que podrían presentarse en el camino.

Dentro de las limitaciones, al ser éste un estudio descriptivo correlacional, se determinó la asociación directa entre las variables motivación y clima organizacional, a pesar de ello, el estudio es relevante debido a que se obtuvo resultados significativos en la asociación de las variables estudiadas.

\section{CONCLUSIONES}

Se concluye que existe asociación entre el clima organizacional y motivación de enfermeras en un hospital de la Policía Nacional del Perú. Así mismo se recomienda realizar estudios posteriores con análisis multivariado para considerar otras variables que podrían estar involucradas en dicha asociación.

\section{Correspondencia}

Ana Sheyla Porras-Balvin

Correo electrónico: anamyle.sh2078@gmail.com

\section{REFERENCIAS BIBLIOGRÁFICAS}

1. Contreras V, Reynaldos K, Cardona L. Clima, ambiente y satisfacción laboral: un desafío para la enfermería. Rev Cubana Enferm. 2015;31(1): 58-69. (Fecha de acceso: 17 de noviembre del 2017) Disponible en: http://scielo.sld.cu/pdf/enf/v31n1/ enf07115.pdf

2. Manosalvas C, Manosalvas L, Nieves J. El clima organizacional y la satisfacción laboral: un análisis cuantitativo riguroso de su relación. AD-minister. 2015;26: 5-15.

3. Ccorimanya D. Motivación y su relación con el clima organizacional de los trabajadores administrativos de la Universidad Nacional José María Arguedas 2015. Tesis de Pregrado. Huancavelica: Universidad Nacional Jose María Arguedas; 2015.
4. Noguera J, Samudio M. Diagnóstico del Clima Organizacional del Hospital Central de las Fuerzas Armadas de Paraguay. Memorias del Inst Investig en Ciencias la Salud. 2014;12(1):15-25.

5. Dos-Santos A, De-Souza R, Cabral A. Clima organizacional em organizações policiais militares Organizational climate in military police organizational. Rev Barasileira Gest e Desenvolv Reg. 2009;5(1):26-70.

6. Policía Nacional del Perú. Proceso de asimilación. Lima: Policía Nacional del Perú; 2017.

7. Palma S. Motivación y clima laboral en personal de entidades universitarias. Rev Investig en Psicol. 2004;3(1):11-21. DOI: https://doi.org/10.15381/ rinvp.v3i1.4909

8. Vasquez M. Factores de la motivación que influyen en el comportamiento organizacional del equipo de salud, Centro Quirúrgico HRDLM, Chiclayo 2011. Tesis de Maestria. Chiclayo: Universidad Catolica Santo Toribio de Mogrovejo; 2013.

9. Sarmiento D. Relación entre clima organizacional y motivación laboral en Sedalib S.A. Tesis de Título Profesional.Trujillo: Universidad Cesar Vallejo; 2015.

10. Paredes E. Motivación personal relacionado al clima organizacional en el Hospital Docente Materno infantil El Carmen. Tesis de Maestria. Huanuco: Universidad Nacional Hermilio Valdizan; 2016.

11. Huaranga I. Clima organizacional de los profesionales de Enfermería del Hospital Municipal. Tesis de Título Profesional. Lima: Universidad Privada San Juan Bautista; 2017 (Fecha de acceso: 17 de noviembre del 2017) Disponible en: http:// repositorio.upsjb.edu.pe/handle/upsjb/532

12. Bernal I, Pedraza N, Sánchez M. El clima organizacional y su relación con la calidad de los servicios públicos de salud: diseño de un modelo teórico. Estudios Gerenciales. 2015; 31 (134): 114-5.

13. Leandro P. Clima Organizacional, Motivación intrínseca y su relación con la satisfacción laboral en trabajadores de diferentes niveles jerárquicos de la Dirección regional de salud. Tesis de Posgrado. Huanuco: Universidad Nacional Hermilio Valdizán; 2016.

14. Rosales F. Motivación y clima laboral del personal de enfermería de un hospital público de Lima. Tesis. Lima: Universidad Cesar Vallejo; 2017.

15. Llagas MG. Motivación y su relación con el desempeño laboral de la Enfermera Servicio de Neonatología Hospital Nacional Arzobispo Loayza, Lima-2015. Tesis. Lima Universidad César Vallejo; 2017; (Fecha de acceso: 17 de noviembre del 2017) Disponible en: http://repositorio.ucv.edu.pe/handle/ $\mathrm{UCV} / 8660$

16. Lucas M. Desempeño de los/las líderes de enfermería y la Motivación del personal en el cumplimiento de 
actividades y la satisfacción de los usuarios del Hospital Fisco Misional Divina Providencia. Tesis de Licenciatura. Esmeraldas, Ecuador: Pontificia Universidad Católica del Ecuador Sede Esmeraldas; 2014.
17. Antonio M, Santiago A. Motivacion y satisfacción laboral del profesional enfermero(a) del Hospital II Ramon Castilla - EsSalud. Tesis de Licenciatura. Lima: Universidad Privada Norbert Wiener; 2015. 